\title{
On the Lucas polynomials and some of their new identities
}

\section{Zhang Jin*}

\section{"Correspondence:}

m18700931869@163.com

School of Information Engineering,

Xi'an University, Xi'an, P.R. China

\section{Abstract \\ The main purpose of this paper is, using the elementary and combination methods, to study the arithmetical properties of the Lucas polynomials and to obtain some new and interesting identities for them.}

MSC: Primary 11B39

Keywords: Fibonacci polynomials; Lucas polynomials; Identity; Elementary method; Combination method

\section{Introduction}

For any non-negative integer $n$, the Fibonacci polynomials $\left\{F_{n}(x)\right\}$ and Lucas polynomials $\left\{L_{n}(x)\right\}$ are defined by the second order linear recursive formulas $F_{n+2}(x)=x F_{n+1}(x)+F_{n}(x)$ and $L_{n+2}(x)=x L_{n+1}(x)+L_{n}(x)$ with $F_{0}(x)=0, F_{1}(x)=1, L_{0}(x)=2$, and $L_{1}(x)=x$. The general terms of $F_{n}(x)$ and $L_{n}(x)$ are given by

$$
F_{n+1}(x)=\sum_{k=0}^{\left[\frac{n}{2}\right]}\left(\begin{array}{c}
n-k \\
k
\end{array}\right) x^{n-2 k}
$$

and

$$
L_{n}(x)=\sum_{k=0}^{\left[\frac{n}{2}\right]} \frac{n}{n-k}\left(\begin{array}{c}
n-k \\
k
\end{array}\right) x^{n-2 k}
$$

where $\left(\begin{array}{l}m \\ n\end{array}\right)=\frac{m !}{n !(m-n) !}$, and $[x]$ denotes the greatest integer $\leq x$.

It is easy to prove the identities

$$
F_{n}(x)=\frac{1}{\sqrt{x^{2}+4}}\left[\left(\frac{x+\sqrt{x^{2}+4}}{2}\right)^{n}-\left(\frac{x-\sqrt{x^{2}+4}}{2}\right)^{n}\right]
$$

and

$$
L_{n}(x)=\left(\frac{x+\sqrt{x^{2}+4}}{2}\right)^{n}+\left(\frac{x-\sqrt{x^{2}+4}}{2}\right)^{n} .
$$
(http://creativecommons.org/licenses/by/4.0/), which permits unrestricted use, distribution, and reproduction in any medium, provided you give appropriate credit to the original author(s) and the source, provide a link to the Creative Commons license, and indicate if changes were made. 
If $x=1$, then $\left\{F_{n}(x)\right\}$ becomes the famous Fibonacci sequences $\left\{F_{n}\right\}$ and $\left\{L_{n}(x)\right\}$ becomes the Lucas sequences $\left\{L_{n}\right\}$.

These sequences and polynomials occupy very important positions in the theory and application of mathematics, so many scholars have studied their various arithmetical properties and obtained a series of important results. For example, Ozeki [1] proved the identity

$$
\sum_{k=1}^{n} F_{2 k}^{2 m+1}=\frac{1}{5^{m}} \sum_{j=0}^{m} \frac{(-1)^{j}}{L_{2 m+1-2 j}}\left(\begin{array}{l}
2 m+1 \\
j
\end{array}\right)\left(F_{(2 m+1-2 j)(2 n+1)}-F_{2 m+1-2 j}\right) .
$$

Prodinger [2] studied the more general summation $\sum_{k=0}^{n} F_{2 k+\delta}^{2 m+1+\epsilon}$, where $\delta, \epsilon \in\{0,1\}$, and obtained many interesting results.

Ma and Zhang [3] used the properties of Chebyshev polynomials to obtain some identities involving Fibonacci numbers and Lucas numbers. Wang and Zhang [4] proved some divisible properties involving Fibonacci numbers and Lucas numbers. Some of other related papers can also be found in references [5-15], here we are not going to list them all.

In this paper, we shall use the elementary and combination methods to study the arithmetical properties of Lucas polynomials, and give some new identities for them. That is, we shall prove the following results.

Theorem 1 For any positive integer $h$ and integer $k \geq 0$, we have

$$
\sum_{n=1}^{h} \frac{L_{2 n(2 k+1)}(x)-2}{n}=\sum_{n=1}^{h} \frac{\left(\begin{array}{c}
h+n \\
h-n
\end{array}\right)}{n} L_{2 k+1}^{2 n}(x) .
$$

Theorem 2 For any integers $h$ and $k \geq 0$, we have

$$
\sum_{n=0}^{h} \frac{L_{(2 n+1)(2 k+1)}(x)}{2 n+1}=\sum_{n=0}^{h} \frac{\left(\begin{array}{c}
h+n+1 \\
h-n
\end{array}\right)}{2 n+1} L_{2 k+1}^{2 n+1}(x) .
$$

Theorem 3 For any integers $n \geq 1$ and $h \geq 0$, we have the identity

$$
\int_{0}^{x} L_{2 h+1}^{2 n}(y) d y=\sum_{k=0}^{n} \frac{(-1)^{n-k}\left(\begin{array}{c}
2 n \\
n-k
\end{array}\right)}{4 k h+2 k+1} L_{4 k h+2 k+1}(x)+\sum_{k=1}^{n} \frac{(-1)^{n-k}\left(\begin{array}{c}
2 n \\
n-k
\end{array}\right)}{4 k h+2 k-1} L_{4 k h+2 k-1}(x) .
$$

Theorem 4 For any integers $n \geq 1$ and $h \geq 0$, we have the identity

$$
\begin{aligned}
\int_{0}^{x} L_{2 h+1}^{2 n+1}(y) d y= & \frac{1}{2} \sum_{k=0}^{n}(-1)^{n-k}\left(\begin{array}{c}
2 n+1 \\
n-k
\end{array}\right)\left(\frac{L_{4 k h+2 k+2 h+2}(x)}{2 k h+k+h+1}+\frac{L_{4 k h+2 k+2 h}(x)}{2 k h+k+h}\right) \\
& -\sum_{k=0}^{n}(-1)^{n-k}\left(\begin{array}{c}
2 n+1 \\
n-k
\end{array}\right)\left(\frac{1}{2 h k+k+h+1}+\frac{1}{2 h k+k+h}\right) .
\end{aligned}
$$

Taking $k=0$, from Theorems 1 and 2 we can deduce the following:

Corollary 1 For any positive integer $h$, we have the identities

$$
\sum_{n=1}^{h} \frac{L_{2 n}(x)-2}{n}=\sum_{n=1}^{h} \frac{\left(\begin{array}{c}
h+n \\
h-n
\end{array}\right)}{n} x^{2 n}
$$


and

$$
\sum_{n=0}^{h} \frac{L_{2 n+1}(x)}{2 n+1}=\sum_{n=0}^{h} \frac{\left(\begin{array}{c}
h+n+1 \\
h-n
\end{array}\right)}{2 n+1} x^{2 n+1}
$$

If $x=1$ and $k=0$, then we also have the following:

Corollary 2 For any positive integer $h$, we have the identities

$$
\sum_{n=1}^{h} \frac{L_{2 n}}{n}=\sum_{n=1}^{h} \frac{\left(\begin{array}{c}
h+n \\
h-n
\end{array}\right)+2}{n} \text { and } \sum_{n=0}^{h} \frac{L_{2 n+1}}{2 n+1}=\sum_{n=0}^{h} \frac{\left(\begin{array}{c}
h+n+1 \\
h-n
\end{array}\right)}{2 n+1}
$$

From Theorems 3 and 4 we can deduce the following corollaries.

Corollary 3 For any integers $n \geq 1$ and $h \geq 0$, we have

$$
\int_{0}^{1} L_{2 h+1}^{2 n}(y) d y=\sum_{k=0}^{n} \frac{(-1)^{n-k}\left(\begin{array}{c}
2 n \\
n-k
\end{array}\right)}{4 k h+2 k+1} L_{4 k h+2 k+1}+\sum_{k=1}^{n} \frac{(-1)^{n-k}\left(\begin{array}{c}
2 n \\
n-k
\end{array}\right)}{4 k h+2 k-1} L_{4 k h+2 k-1} .
$$

Corollary 4 For any integers $n \geq 1$ and $h \geq 0$, we have

$$
\int_{0}^{1} L_{2 h+1}^{2 n+1}(y) d y=\sum_{k=0}^{n} \frac{(-1)^{n-k}}{2}\left(\begin{array}{c}
2 n+1 \\
n-k
\end{array}\right)\left(\frac{L_{4 k h+2 k+2 h+2}-2}{2 k h+k+h+1}+\frac{L_{4 k h+2 k+2 h}-2}{2 k h+k+h}\right) .
$$

\section{Several simple lemmas}

Lemma 1 For any positive integers $n$, we have the identities

$$
\begin{aligned}
& \int_{0}^{x} L_{2 n}(y) d y=\frac{L_{2 n+1}(x)}{2 n+1}+\frac{L_{2 n-1}(x)}{2 n-1} \\
& \int_{0}^{x} L_{2 n+1}(y) d y=\frac{L_{2 n+2}(x)}{2 n+2}+\frac{L_{2 n}(x)}{2 n}-\frac{2 n+1}{n(n+1)}
\end{aligned}
$$

Proof Note the identities $\left(x+\sqrt{x^{2}+4}\right)^{\prime}=1+\frac{x}{\sqrt{x^{2}+4}}=\frac{x+\sqrt{x^{2}+4}}{\sqrt{x^{2}+4}}$ and $\left(x-\sqrt{x^{2}+4}\right)^{\prime}=1-$ $\frac{x}{\sqrt{x^{2}+4}}=-\frac{x-\sqrt{x^{2}+4}}{\sqrt{x^{2}+4}}$. From the definitions of the polynomials $F_{n}(x)$ and $L_{n}(x)$, we have

$$
\begin{aligned}
L_{n}^{\prime}(x) & =\frac{n}{\sqrt{x^{2}+4}}\left(\frac{x+\sqrt{x^{2}+4}}{2}\right)^{n}-\frac{n}{\sqrt{x^{2}+4}}\left(\frac{x-\sqrt{x^{2}+4}}{2}\right)^{n} \\
& =n \cdot F_{n-1}(x) .
\end{aligned}
$$

Applying (3), the integration by parts, and the recursive formulae of $L_{n}(x)$ and $F_{n}(x)$, we have

$$
\begin{aligned}
\int_{0}^{x} L_{n}(y) d y & =x L_{n}(x)-\int_{0}^{x} y L_{n}^{\prime}(y) d y \\
& =x L_{n}(x)-n \int_{0}^{x} y F_{n-1}(y) d y \\
& =L_{n+1}(x)-L_{n-1}(x)-n \int_{0}^{x}\left(F_{n}(y)-F_{n-2}(y)\right) d y
\end{aligned}
$$




$$
\begin{aligned}
& =L_{n+1}(x)-L_{n-1}(x)-\frac{n}{n+1}\left(L_{n+1}(x)-L_{n+1}(0)\right)+\frac{n}{n-1}\left(L_{n-1}(x)-L_{n-1}(0)\right) \\
& =\frac{L_{n+1}(x)}{n+1}+\frac{L_{n-1}(x)}{n-1}+\frac{n L_{n+1}(0)}{n+1}-\frac{n L_{n-1}(0)}{n-1}
\end{aligned}
$$

If $n=2 k$, then note that $L_{2 k+1}(0)=L_{2 k-1}(0)=0$. From (4) we have

$$
\int_{0}^{x} L_{2 k}(y) d y=\frac{L_{2 k+1}(x)}{2 k+1}+\frac{L_{2 k-1}(x)}{2 k-1} .
$$

If $n=2 k+1$, then by (2) we have $L_{2 k+2}(0)=L_{2 k}(0)=2$. From (4) we have

$$
\int_{0}^{x} L_{2 k+1}(y) d y=\frac{L_{2 k+2}(x)}{2 k+1}+\frac{L_{2 k}(x)}{2 k}-\frac{2 k+1}{k(k+1)} .
$$

Now Lemma 1 follows from (5) and (6).

Lemma 2 For any positive integer $n$ and non-negative integer $k$, we have the identity

$$
L_{n}\left(L_{2 k+1}(x)\right)=L_{n(2 k+1)}(x) .
$$

Proof Let $\alpha=\frac{x+\sqrt{x^{2}+4}}{2}$ and $\beta=\frac{x-\sqrt{x^{2}+4}}{2}$. Then replace $x$ by $L_{2 k+1}(x)$ in (2) and note that $\alpha^{2 k+1} \beta^{2 k+1}=-1$, we have

$$
\begin{aligned}
L_{2 k+1}(x)+\sqrt{L_{2 k+1}^{2}(x)+4} & =\alpha^{2 k+1}+\beta^{2 k+1}+\sqrt{\left(\alpha^{2 k+1}+\beta^{2 k+1}\right)^{2}+4} \\
& =\alpha^{2 k+1}+\beta^{2 k+1}+\sqrt{\alpha^{2(2 k+1)}+\beta^{2(2 k+1)}-2+4} \\
& =\alpha^{2 k+1}+\beta^{2 k+1}+\sqrt{\left(\alpha^{2 k+1}-\beta^{2 k+1}\right)^{2}}=2 \alpha^{2 k+1}
\end{aligned}
$$

and

$$
\begin{aligned}
L_{2 k+1}(x)-\sqrt{L_{2 k+1}^{2}(x)+4} & =\alpha^{2 k+1}+\beta^{2 k+1}-\sqrt{\left(\alpha^{2 k+1}+\beta^{2 k+1}\right)^{2}+4} \\
& =\alpha^{2 k+1}+\beta^{2 k+1}-\sqrt{\left(\alpha^{2 k+1}-\beta^{2 k+1}\right)^{2}}=2 \beta^{2 k+1} .
\end{aligned}
$$

From (2) we have the identity

$$
\begin{aligned}
L_{n}\left(L_{2 k+1}(x)\right) & =\left(\frac{L_{2 k+1}+\sqrt{L_{2 k+1}^{2}+4}}{2}\right)^{n}+\left(\frac{L_{2 k+1}-\sqrt{L_{2 k+1}^{2}+4}}{2}\right)^{n} \\
& =\alpha^{n(2 k+1)}+\beta^{n(2 k+1)}=L_{n(2 k+1)}(x) .
\end{aligned}
$$

This proves Lemma 2.

Lemma 3 For any non-negative integer $n$, we have the identities

$$
x^{2 n}=\frac{(-1)^{n}}{2}\left(\begin{array}{c}
2 n \\
n
\end{array}\right) \cdot L_{0}(x)+\sum_{k=1}^{n}(-1)^{n-k}\left(\begin{array}{c}
2 n \\
n-k
\end{array}\right) \cdot L_{2 k}(x)
$$


and

$$
x^{2 n+1}=\sum_{k=0}^{n}(-1)^{n-k}\left(\begin{array}{c}
2 n+1 \\
n-k
\end{array}\right) \cdot L_{2 k+1}(x)
$$

Proof From the definition of $L_{n}(x)$ we know that $L_{2 k}(x)$ is an even function. So we may suppose that

$$
x^{2 n}=\sum_{k=0}^{n} a_{k} \cdot L_{2 k}(x)
$$

Taking $x=2 i \cos \theta$ in (7) and noting that $x^{2}+4=4-4 \cos ^{2} \theta=4 \sin ^{2} \theta$, from Euler's formula we have

$$
\begin{aligned}
L_{2 k}(2 i \cos \theta) & =\left(\frac{2 i \cos \theta+\sqrt{4 \sin ^{2} \theta}}{2}\right)^{2 k}+\left(\frac{2 i \cos \theta-\sqrt{4 \sin ^{2} \theta}}{2}\right)^{2 k} \\
& =(i \cos \theta+\sin \theta)^{2 k}+(i \cos \theta-\sin \theta)^{2 k} \\
& =(-1)^{k}(\cos \theta-i \sin \theta)^{2 k}+(-1)^{k}(\cos \theta+i \sin \theta)^{2 k} \\
& =(-1)^{k} \cdot 2 \cdot \cos (2 k \theta) .
\end{aligned}
$$

Then from (7) and (8) we have

$$
(-1)^{n} 4^{n} \cos ^{2 n} \theta=\sum_{k=0}^{n} a_{k} \cdot L_{2 k}(2 i \cos \theta)=2 \sum_{k=0}^{n} a_{k} \cdot(-1)^{k} \cos (2 k \theta) .
$$

Note the identities

$$
\int_{0}^{\pi} 2 \cos (m \theta) \cos (n \theta) d \theta= \begin{cases}\pi, & \text { if } m=n \neq 0 \\ 0, & \text { if } m \neq n \\ 2 \pi, & \text { if } m=n=0\end{cases}
$$

and

$$
\int_{0}^{\pi} \cos ^{2 n}(\theta) \cos (2 k \theta) d \theta=\pi \cdot \frac{(2 n) !}{(2 n-2 k) ! !(2 n+2 k) ! !}=\frac{\pi}{4^{n}} \cdot\left(\begin{array}{c}
2 n \\
n-k
\end{array}\right) .
$$

From (9) and (10) we have

$$
a_{k} \cdot(-1)^{k} \pi=(-1)^{n} 4^{n} \int_{0}^{\pi} \cos ^{2 n}(\theta) \cos (2 k \theta) d \theta=(-1)^{n} \pi \cdot\left(\begin{array}{c}
2 n \\
n-k
\end{array}\right)
$$

or

$$
a_{0}=\frac{(-1)^{n}}{2} \cdot\left(\begin{array}{c}
2 n \\
n
\end{array}\right) \quad \text { and } \quad a_{k}=(-1)^{n-k} \cdot\left(\begin{array}{c}
2 n \\
n-k
\end{array}\right), \quad 1 \leq k \leq n .
$$

Combining (7), (9), and (11), we can deduce the first identity of Lemma 3. 
Similarly, since $L_{2 k+1}(x)$ is an odd function, we can suppose that

$$
x^{2 n+1}=\sum_{k=0}^{n} b_{k} \cdot L_{2 k+1}(x)
$$

Taking $x=2 i \cos \theta$ in (12) and noting that

$$
\begin{aligned}
L_{2 k}(2 i \sin \theta) & =\left(\frac{2 i \sin \theta+\sqrt{4 \cos ^{2} \theta}}{2}\right)^{2 k}+\left(\frac{2 i \sin \theta-\sqrt{4 \cos ^{2} \theta}}{2}\right)^{2 k} \\
& =(i \sin \theta+\cos \theta)^{2 k}+(i \sin \theta-\cos \theta)^{2 k} \\
& =(\cos \theta+i \sin \theta)^{2 k}+(\cos \theta-i \sin \theta)^{2 k} \\
& =\cos (2 k \theta)+i \sin (2 k \theta)+\cos (2 k \theta)-i \sin (2 k \theta) \\
& =2 \cdot \cos (2 k \theta),
\end{aligned}
$$

we have

$$
(-1)^{n} 4^{n} \cos ^{2 n+1} \theta=\sum_{k=0}^{n} b_{k} \cdot(-1)^{k} \cos ((2 k+1) \theta)
$$

From (10) and (13) we may immediately deduce that

$$
b_{k}=\frac{2(-1)^{n-k}}{\pi} \cdot 4^{n} \cdot \int_{0}^{\pi} \cos ^{2 n+1}(\theta) \cos ((2 k+1) \theta) d \theta=(-1)^{n-k}\left(\begin{array}{c}
2 n+1 \\
n-k
\end{array}\right) .
$$

Now the second identity of Lemma 3 follows from (12) and (14).

\section{Proofs of the theorems}

Using the lemmas in Sect. 2, we can prove our theorems easily. First we prove Theorem 2. Similarly, we can also deduce Theorem 1 and then omit its proving process here. From Lemma 1 and the definition of $L_{n}(x)$, we have

$$
\begin{aligned}
\sum_{n=0}^{h} L_{2 n}(x) & =\sum_{n=0}^{h}\left(\alpha^{2 n}+\beta^{2 n}\right) \\
& =\frac{\alpha^{2 h+2}-1}{\alpha^{2}-1}+\frac{\beta^{2 h+2}-1}{\beta^{2}-1} \\
& =\frac{L_{2 h+2}(x)-L_{2 h}(x)}{L_{2}(x)-2}+1 \\
& =\frac{L_{2 h+1}(x)}{x}+1
\end{aligned}
$$

Applying (15) and Lemma 1, we have

$$
\int_{0}^{x} \sum_{n=1}^{h} L_{2 n}(y) d y=\int_{0}^{x}\left(\frac{L_{2 h+1}(y)}{y}-1\right) d y=\sum_{n=1}^{h}\left(\frac{L_{2 n+1}(x)}{2 n+1}+\frac{L_{2 n-1}(x)}{2 n-1}\right)
$$


or

$$
\begin{aligned}
2 \sum_{n=0}^{h} \frac{L_{2 n+1}(x)}{2 n+1}= & \int_{0}^{x} \frac{L_{2 h+1}(y)}{y} d y+\frac{L_{2 h+1}(x)}{2 h+1} \\
= & \sum_{k=0}^{h} \frac{2 h+1}{2 h+1-k}\left(\begin{array}{c}
2 h+1-k \\
k
\end{array}\right) \int_{0}^{x} y^{2 h-2 k} d y \\
& +\frac{1}{2 h+1} \sum_{k=0}^{h} \frac{2 h+1}{2 h+1-k}\left(\begin{array}{c}
2 h+1-k \\
k
\end{array}\right) x^{2 h+1-2 k} \\
= & \sum_{k=0}^{h} \frac{2}{2 h+1-2 k}\left(\begin{array}{c}
2 h+1-k \\
k
\end{array}\right) \cdot x^{2 h+1-2 k} \\
= & 2 \sum_{n=0}^{h} \frac{\left(\begin{array}{c}
h+n+1 \\
h-n
\end{array}\right)}{2 n+1} x^{2 n+1} .
\end{aligned}
$$

Now Theorem 2 follows from (16) and Lemma 2 with $x=L_{2 k+1}(y)$.

To prove Theorem 3, taking $x=L_{2 h+1}(y)$ in Lemma 3, we have

$$
L_{2 h+1}^{2 n}(y)=(-1)^{n}\left(\begin{array}{c}
2 n \\
n
\end{array}\right)+\sum_{k=1}^{n}(-1)^{n-k}\left(\begin{array}{c}
2 n \\
n-k
\end{array}\right) \cdot L_{2 k(2 h+1)}(y) .
$$

Integrating for $y$ from 0 to $x$ in (17), then applying Lemma 1, we may immediately deduce

$$
\int_{0}^{x} L_{2 h+1}^{2 n}(y) d y=\sum_{k=0}^{n} \frac{(-1)^{n-k}\left(\begin{array}{c}
2 n \\
n-k
\end{array}\right)}{4 k h+2 k+1} L_{4 k h+2 k+1}(x)+\sum_{k=1}^{n} \frac{(-1)^{n-k}\left(\begin{array}{c}
2 n \\
n-k
\end{array}\right)}{4 k h+2 k-1} L_{4 k h+2 k-1}(x) .
$$

This proves Theorem 3.

Similarly, we can also deduce Theorem 4.

\section{Acknowledgements}

The author would like to thank the referees for their very helpful and detailed comments, which have significantly improved the presentation of this paper. This work is supported by the N.S.F. (Grant No. 11771351) of P.R. China.

\section{Competing interests}

The author declares that she has no competing interests.

\section{Authors' contributions}

All authors read and approved the final manuscript.

\section{Publisher's Note}

Springer Nature remains neutral with regard to jurisdictional claims in published maps and institutional affiliations.

Received: 9 January 2018 Accepted: 15 February 2018 Published online: 06 April 2018

\section{References}

1. Ozeki, K.: On Melham's sum. Fibonacci Q. 46/47, 107-110 (2008/2009)

2. Prodinger, H.: On a sum of Melham and its variants. Fibonacci Q. 46/47, 207-215 (2008/2009)

3. Ma, R., Zhang, W.: Several identities involving the Fibonacci numbers and Lucas numbers. Fibonacci Q. 45, 164-170 (2007)

4. Wang, T., Zhang, W.: Some identities involving Fibonacci. Lucas polynomials and their applications. Bull. Math. Soc. Sci. Math. Roum. 55, 95-103 (2012)

5. Melham, R.S.: Some conjectures concerning sums of odd powers of Fibonacci and Lucas numbers. Fibonacci Q. 46/47, 312-315 (2008/2009) 
6. Li, X.: Some identities involving Chebyshev polynomials. Math. Probl. Eng. 2015, Article ID 950695 (2015)

7. Ma, Y., Lv, X.: Several identities involving the reciprocal sums of Chebyshev polynomials. Math. Probl. Eng. 2017, Article ID 4194579 (2017)

8. Kim, D.S., Dolgy, D.V., Kim, T., Rim, S.H.: Identities involving Bernoulli and Euler polynomials arising from Chebyshev polynomials. Proc. Jangjeon Math. Soc. 15, 361-370 (2012)

9. Kim, D.S., Kim, T., Lee, S.: Some identities for Bernoulli polynomials involving Chebyshev polynomials. J. Comput. Anal. Appl. 16, 172-180 (2014)

10. Kim, T., Kim, D.S., Seo, J.J., Dolgy, D.V.: Some identities of Chebyshev polynomials arising from non-linear differential equations. J. Comput. Anal. Appl. 23, 820-832 (2017)

11. He, Y.: Some new results on products of Apostol-Bernoulli and Apostol-Euler polynomials. J. Math. Anal. Appl. 431 34-46 (2015)

12. He, Y., Wang, C.P.: New symmetric identities involving the Eulerian polynomials. J. Comput. Anal. Appl. 17, 498-504 (2014)

13. He, Y., Kim, D.S.: General convolution identities for Apostol-Bernoulli, Euler and Genocchi polynomials. J. Nonlinear Sci. Appl. 9, 4780-4797 (2016)

14. Chen, L., Zhang, W.: Chebyshev polynomials and their some interesting applications. Adv. Differ. Equ. 2017, 303 (2017)

15. Yi, Y., Zhang, W.: Some identities involving the Fibonacci polynomials. Fibonacci Q. 40, 314-318 (2002)

\section{Submit your manuscript to a SpringerOpen ${ }^{\circ}$ journal and benefit from:}

- Convenient online submission

- Rigorous peer review

- Open access: articles freely available online

- High visibility within the field

- Retaining the copyright to your article

Submit your next manuscript at $\gg$ springeropen.com 